\title{
Depressão em Graduandos de Enfermagem
}

\author{
Depression among Nursing Undergraduate Students \\ Depresión en Graduandos de Enfermería
}

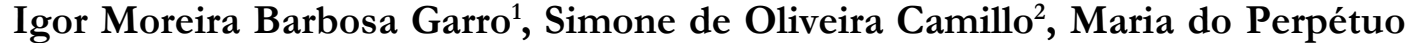 Socorro de Sousa Nóbrega ${ }^{3}$

\section{RESUMO}

Objetivo: a proposta deste artigo foi a de averiguar o número de graduandos de Enfermagem da Faculdade de Medicina do ABC que se apresentam com sintomas indicativos de depressão. Métodos: estudo exploratório e o instrumento utilizado foi o Inventário de Depressão de Beck. A população foi constituída de 119 discentes. Resultados: apontam que a população estudada merece significativa atenção uma vez que, dos 119 alunos do curso de graduação em enfermagem, 33 (26,06\%) apresentaram sintomas indicativos de depressão. Estes números são importantes, pois demonstram que essa população vivencia situações conflituosas no dia-a-dia, podendo ser estas responsáveis pelo desencadeamento de sintomas indicativos de depressão. Conclusão: é de grande relevância mostrar a necessidade da criação de programas de suporte psicológico aos alunos de graduação em enfermagem de todas as séries, objetivando oferecer suporte para lidarem com as situações de sofrimento psíquico.

DESCRITORES: Depressão; Estudante de enfermagem; Saúde mental

\begin{abstract}
Objective: to explore depressive episodes among nursing undergraduate students from the School of Nursing in the College of Medicine of ABC Methods: this was a descriptive exploratory study. The Beck Depression Inventory was used to measure depressive episodes. One hundred and nineteen undergraduate nursing students participated in this study. Results: thirty three $(22.06 \%)$ of nursing undergraduate students reported depressive episodes. Conclusion: this is an important finding because nursing undergraduate students may need to have more psycho-social support to prepare them to cope with stressful patient situations.
\end{abstract}

Keywords: Depression; Students nursing; Mental health

\section{RESUMEN}

Objetivo: la propuesta en este artículo fue averiguar el número de graduandos de Enfermería, de la Facultad de Medicina del ABC, que se presentan con síntomas de depresión. Métodos: estudio exploratorio cuyo instrumento utilizado fue el Inventario de Depresión de Beck. La población estuvo constituida por 119 discentes. Resultados: la población estudiada merece significativa atención pues, de los 119 alumnos del pregrado en enfermería, el 26,06\% (33) presentaron síntomas indicativos de depresión. Estos números son importantes, debido a que demuestran que esa población vivencia situaciones conflictivas diariamente, pudiendo ser responsables del desencadenamiento de los síntomas de depresión. Conclusión: es relevante mostrar la necesidad de crear programas de soporte psicológico a los alumnos de pregrado en enfermería a lo largo de su formación profesional, con el objetivo de ofrecerle soporte para lidiar con las situaciones de sufrimiento psíquico.

DESCIPTORS: Depresión; Estudiantes de enfermería; Salud mental

\footnotetext{
${ }^{1}$ Enfermeiro, graduado pelo Curso de Enfermagem da Faculdade de Medicina da Fundação do ABC Santo André. São Paulo (SP), Brasil.

${ }^{2}$ Mestre em Ciências pelo Instituto de Saúde da Secretaria do Estado da Saúde de São Paulo. Professora do Curso de Enfermagem da Faculdade de Medicina da Fundação do ABC, Santo André. São Paulo (SP), Brasil.

${ }^{3}$ Doutoranda em Saúde do Adulto. Professora Substituta do Departamento de Enfermagem da Universidade Federal de São Paulo -UNIFESP-e do Curso de Enfermagem da Faculdade de Medicina da Fundação do ABC, Santo André, São Paulo (SP), Brasil.
} 


\section{INTRODUÇÃO}

A depressão é um dos Transtornos do Humor, caracterizada por lentificação dos processos psíquicos, humor depressivo e/ou irritável, redução da energia (desânimo, cansaço fácil), incapacidade parcial ou total de sentir alegria e/ou prazer, desinteresse, apatia ou agitação psicomotora, dificuldade de concentração, pensamentos de cunho negativo, com perda da capacidade de planejar o futuro e alteração do juízo da realidade ${ }^{(1)}$. Os sintomas depressivos podem ter duração variada (episódios únicos, recorrentes, crônicos ou breves) e com intensidades diferentes (comprometimento no funcionamento do dia-a-dia de forma leve, moderada ou grave $)^{(2)}$.

Muitas pessoas confundem depressão com tristeza, luto ou uma "fase ruim", e a atribuem às dificuldades cotidianas ou mesmo ao estresse. A depressão pode aparecer como uma resposta a situações reais, devido a uma reação vivencial depressiva, quando diante de fatos desagradáveis, aborrecedores, frustrações e perdas. Tratase, neste caso, de uma resposta a conflitos íntimos e determinados por fatores vivenciais. Pode aparecer, ainda, acompanhada ou aparentemente motivada, por situações anímicas, onde certas perspectivas futuras, anseios e objetivos de vida estão representados intrapsiquicamente de maneira negativa ${ }^{(3)}$.

Atualmente, a depressão é considerada um problema de saúde tão freqüente quanto diabetes e hipertensão. Em relação à sua freqüência, estima-se que de $15 \%$ a $25 \%$ da população geral sofre deste transtorno, ou seja, a cada quatro pessoas uma irá fazer tratamento para esse quadro, pelo menos alguma vez na vida ${ }^{(4)}$. No Brasil, aproximadamente 24 a 30 milhões de pessoas apresentam, apresentaram ou virão a ter pelo menos um episódio depressivo ao longo da vida. Pessoas de ambos os sexos, de todas as raças etnias, idades e condições sociais podem ser acometidas, tendo nas mulheres o grupo mais vulnerável, na proporção de duas a três para cada homem ${ }^{(3,5)}$.

Em relação à idade, freqüentemente a depressão acomete os adultos em fase produtiva, além de crianças, adolescentes e idosos. No adolescente os sintomas depressivos são semelhantes aos apresentados pelos adultos, entretanto quadros de raiva, apatia, comportamento agressivo, queda no rendimento escolar podem também estar presentes ${ }^{(2)}$. Quanto ao tratamento, indica-se a exclusão de doenças orgânicas, que podem se confundir com depressão ou piorar o episódio depressivo, e o mesmo é feito com medicamentos, eletroconvulsoterapia e psicoterapia (individual ou em grupo) $)^{(6)}$.

Uma população que merece atenção, por estar em contato com o sofrimento psíquico, são os estudantes e profissionais da área da saúde. Estes são marcados constantemente por incertezas, ansiedades, que devem ser cuidadosamente consideradas, uma vez que ao serem vivenciadas, revelam os próprios sentimentos, como também a dificuldade em manejá-los ${ }^{(7)}$. Essas situações provocam uma reação de choque entre o "bom" e o "ruim", gerada principalmente pela angústia decorrente da inadaptação do indivíduo frente a uma nova situação ${ }^{(8-10)}$.

Os estudos encontrados referentes à depressão e os demais sofrimentos psíquicos (prevalência de suicídio, uso de drogas, distúrbios conjugais e disfunções profissionais) concentram-se, em sua grande maioria, nos médicos e estudantes de medicina ${ }^{(3)}$. Quanto aos graduandos de Enfermagem, os estudos estão centrados em sua maioria na descrição de sentimentos que são desencadeados durante o processo ensino-aprendizagem prático ${ }^{(7,11)}$.

A experiência vivida no estágio pelos graduandos de Enfermagem, concomitantemente com a realidade individual, gera uma situação ameaçadora, a ponto de mantê-los alienados, e contribuir para emergir o sentimento de fuga. Porém, outros conseguem adequarse a essa situação, sendo capazes de aproveitar as oportunidades oferecidas. Os acadêmicos deixam explícitos sentimentos, como insegurança e medo, quando percebem que terão que agir junto ao paciente com a postura de um profissional. Esses sentimentos justificamse pela dificuldade na interação e na compreensão da comunicação paciente-aluno, cuja preocupação maior é a sensação de prejuízo que pode ser causado ao paciente, por suas inabilidades e conhecimentos ainda limitados ${ }^{(9)}$.

No decorrer do estágio os acadêmicos de enfermagem vivenciam momentos de dificuldade pessoal e interpessoal. Ao buscarem recursos de manejo baseados em experiências anteriores, a concepção da situação tende a mudar, levando a um ajustamento e adaptação a esse momento. Com o passar do tempo e o amadurecimento emocional, os momentos conflitantes tornam-se menos freqüentes e mais simples ${ }^{(12)}$.

Sabemos que a formação acadêmica do graduando de enfermagem é um processo de desenvolvimento, no qual o aluno deve aprender a lidar: com sentimentos de vulnerabilidade; com o gerenciamento do crescente volume de informações; com o planejamento da carreira profissional; com o estresse decorrente de certas características dos estágios práticos (fadiga, pacientes dificeis); com problemas relativos à qualidade do ensino e ao ambiente educacional; com o estresse que está vinculado a características individuais e situacões pessoais (vulnerabilidades psicológicas, situação socioeconômica, problemas familiares, situações estressantes representadas pela busca de independência e autonomia em relação aos pais, conflitos entre os trabalhos acadêmicos e lazer, conflitos ligados aos relacionamentos afetivos), além do desgaste ligado ao contato com pessoas doentes e com a morte.

Diante da relevância desta problemática, pretendeuse com este estudo fazer um levantamento dos sintomas indicativos de depressão em alunos de um curso de graduação em Enfermagem, objetivando oferecer 
subsídios para medidas preventivas e possíveis projetos de apoio.

\section{MÉTODOS}

Este estudo foi aprovado pelo Comitê de Ética e Pesquisa com Seres Humanos da Faculdade de Medicina do ABC, respeitando a Resolução n ${ }^{\circ}$ 196/96 e somente após orientação sobre os objetivos e assinatura do Termo de Consentimento Livre e Esclarecido junto aos sujeitos da pesquisa, a coleta de dados foi iniciada. Esta ocorreu durante o mês de agosto de 2004, e participaram 119 alunos dos 150 atualmente matriculados, nas quatro séries do Curso de Graduação em Enfermagem da Faculdade de Medicina da Fundação ABC, situada no município de Santo André-São Paulo.

Trata-se de um estudo exploratório-descritivo, cujo instrumento utilizado foi o Inventário de Depressão de Beck (Anexo) traduzido para vários idiomas e validado em diferentes países ${ }^{13}$. O Inventário de Beck trabalha com uma amplitude de itens para avaliar depressão, é auto - aplicável, usado tanto em pesquisa como na prática clínica ${ }^{(14)}$.

O Inventário de Depressão de Beck consiste de 21 itens, incluindo sintomas e atitudes, cuja intensidade varia de 0 a 3. Referem-se à tristeza, pessimismo, sensação de fracasso, falta de satisfação, sensação de culpa, sensação de punição, autodepreciação, auto-acusações, idéias suicidas, crises de choro, irritabilidade, retraşão social, indecisão, distorção da imagem corporal, inibição para o trabalho, distúrbio do sono, fadiga, perda de apetite, perda de peso, preocupação somática, diminuição de libido ${ }^{(14)}$.

A escolha do ponto de corte depende da natureza da amostra e dos objetivos do estudo. Para amostras de pacientes com diagnóstico médico de transtorno afetivo o "Center for Cognitive Therapy" recomenda utilizar o Inventário de Depressão de Beck para classificar o nível, sob os seguintes pontos de corte: menor que $10=\mathrm{sem}$ depressão ou depressão mínima; 10 a 18 = depressão de leve a moderada; 19 a 29 = depressão moderada a grave; de 30 a $63=$ depressão grave ${ }^{(13)}$.

Já para amostras sem diagnóstico médico de transtorno afetivo (caso dos sujeitos deste estudo), as diretrizes são diferentes: escores superiores a 15 indicam disforia (mudança repentina e transitória do estado de ânimo). Os sintomas indicativos de depressão são definidos com escores superiores a 20, preferencialmente com diagnóstico clínico concomitante ${ }^{(15-16)}$.

O tratamento estatístico definido para análise dos dados foi o percentual simples. Os resultados foram apresentados por meio de tabelas, com discussão posterior baseada em literatura científica.

\section{RESULTADOS}

Tabela 1 - Caracterização da idade por faixa etária e sexo dos alunos do Curso de Graduação de Enfermagem da Faculdade de Medicina da Fundação ABC. Santo André, 2004.

\begin{tabular}{|c|c|c|c|c|c|c|c|c|}
\hline \multirow{2}{*}{\multicolumn{3}{|c|}{$\begin{array}{c}\text { IDADE } \\
\text { (Faixa } \\
\text { etárias) }\end{array}$}} & \multirow{2}{*}{\multicolumn{2}{|c|}{ MASCULINO }} & \multirow{2}{*}{\multicolumn{2}{|c|}{$\begin{array}{l}\text { O } \\
\text { FEMININO }\end{array}$}} & \multirow{2}{*}{\multicolumn{2}{|c|}{ TOTAL }} \\
\hline & & & & & & & & \\
\hline & & & $\mathbf{N}$ & $\%$ & $\mathbf{N}$ & $\%$ & $\mathbf{N}$ & $\%$ \\
\hline 17 & - & 20 & 1 & 0,84 & 52 & 43,69 & 53 & 44,56 \\
\hline 21 & -1 & 23 & 5 & 4,20 & 33 & 27,75 & 38 & 31,94 \\
\hline 24 & - & 26 & 2 & 1,68 & 10 & 8,40 & 12 & 10,08 \\
\hline 27 & $1-1$ & 30 & 1 & 0,84 & 6 & 5,04 & 07 & 5,88 \\
\hline 31 & -1 & 33 & - & - & 4 & 3,36 & 04 & 3,36 \\
\hline 34 & - & 40 & - & - & 5 & 4,20 & 05 & 4,20 \\
\hline \multicolumn{3}{|c|}{ TOTAL } & 9 & 7,56 & 110 & 92,44 & 119 & 100,00 \\
\hline
\end{tabular}

$\mathrm{Na}$ Tabela 1 observa-se que houve predominância de estudantes do sexo feminino (92,44\%). Com relação à idade, a amostra encontra-se, de forma predominante, na faixa etária de 17 a 20 anos, representando o final da adolescência, ou seja, jovens com pouca experiência de vida que elegeram a enfermagem como profissão, sem, muitas vezes, terem clara a idéia do que isso representa ou significa para suas vidas.

Tabela 2- Distribuição das faixas de sintomas indicativos de depressão de estudantes de Enfermagem de primeira à quarta séries submetidos ao Inventário de Depressão de Beck. Santo André, 2004.

\begin{tabular}{lcc}
\hline \multicolumn{1}{c}{ ALUNOS DA 1 ${ }^{\mathbf{a}} \mathbf{A} 4^{\text {a SÉRIE }}$} & N & \% \\
\hline${\text { Menor } \mathrm{n}^{\text {o }} \text { de pontos indicativos de sintomas de }}^{33}$ & 26,06 \\
Depressão $($ escores > 20) & & \\
Maior $\mathrm{n}^{\mathrm{o}}$ de pontos indicativos de sintomas de & 86 & 73,94 \\
Depressão $($ escores < 20) & & \\
\hline TOTAL & $\mathbf{1 1 9}$ & $\mathbf{1 0 0 , 0 0}$ \\
\hline
\end{tabular}

Na Tabela 2 pode-se observar que do total de 119 alunos do Curso de Graduação em Enfermagem, de todas as séries, $33(26,06 \%)$ apresentaram escores $>20$ para sintomas indicativos de depressão no Inventário de Depressão de Beck e $86(73,94 \%)$ alunos não apresentaram.

$\mathrm{Na}$ Tabela 3 verifica-se que dos 39 alunos do primeiro ano de Enfermagem, 7 apresentaram escores $>20$ para sintomas indicativos de depressão. $\mathrm{Na}$ segunda série do Curso de Graduação em Enfermagem, dos 38 alunos respondentes $13(34,21 \%)$ encontram-se com escores > 20 para sintomas indicativos de depressão. Dos 23 alunos do terceiro ano de Graduação de Enfermagem 21,73\% apresentam escores $>20$ para sintomas indicativos de depressão, deste grupo 5 alunos são do sexo feminino. Em relação aos 19 alunos da quarta série de Graduação em Enfermagem, 08 (42,10\%) apresentam escores $>20$ para sintomas indicativos de depressão.

Dos 31 estudantes que trabalham na área da saúde 09 
Tabela 3 - Distribuição das faixas de sintomas indicativos de depressão de estudantes de Enfermagem submetidos ao Inventário de Depressão de Beck por série. Santo André, 2004.

\begin{tabular}{|c|c|c|c|c|c|c|c|c|}
\hline \multirow{2}{*}{$\begin{array}{l}\text { FAIXA DE } \\
\text { SINTOMAS }\end{array}$} & \multicolumn{2}{|c|}{ 1'SÉRIE } & \multicolumn{2}{|c|}{$2^{\circ}$ SÉRIE } & \multicolumn{2}{|c|}{$3^{\circ}$ SÉRIE } & \multicolumn{2}{|c|}{$4^{\circ}$ SÉRIE } \\
\hline & $\mathbf{N}$ & $\%$ & $\mathbf{N}$ & $\%$ & $\mathbf{N}$ & $\%$ & $\mathbf{N}$ & $\%$ \\
\hline $\begin{array}{l}\text { Menor } \mathrm{n}^{\circ} \text { de pontos } \\
\text { indicativos de sintomas de } \\
\text { depressão } \\
(\text { escores }>20)\end{array}$ & 7 & 17,95 & 13 & 34,21 & 5 & 21,73 & 8 & 42,10 \\
\hline $\begin{array}{l}\text { Maior } \mathrm{n}^{\circ} \text { de pontos } \\
\text { indicativos de sintomas de } \\
\text { depressão } \\
(\text { escores }<20)\end{array}$ & 32 & 82,05 & 25 & 65,79 & 18 & 78,27 & 11 & 57,90 \\
\hline TOTAL & 39 & 100,00 & 38 & 100,00 & 23 & 100,00 & 19 & 100,0 \\
\hline
\end{tabular}

$(29,04 \%)$ apresentam escores $>20$ para sintomas indicativos de depressão.

\section{DISCUSSÃO}

Alicerçando-se na história da Enfermagem, justificase um maior número de pessoas do sexo feminino neste curso $^{(17)}$. Ao comparar os alunos da primeira à quarta série, observa-se que os índices maiores de alunos com escores $>20$ para sintomas indicativos de depressão, são da segunda $(34,21 \%)$ e quarta $(42,10 \%)$ séries. Ao passo que a primeira e terceira séries apresentaram um índice menor de sintomas indicativos de depressão de 17,95\% e $21,73 \%$, respectivamente.

Ao recorrer à literatura, no intuito de entender o elevado número de alunos com sintomas indicativos de depressão na segunda e quarta séries, deparou-se com explicações de alguns estudiosos ${ }^{(8-10,18)}$ a respeito da temática em estudantes que cursam a graduação em Enfermagem. O primeiro contato do aluno com o paciente em estágio prático no hospital ocorre durante a segunda série de graduação. Neste ano ministra-se a disciplina básica de Semiologia e Semiotécnica, que familiariza os acadêmicos com os procedimentos de enfermagem, manejo de materiais, ambientes hospitalares e com os princípios do processo de trabalho na enfermagem. Esta disciplina, teórico-prática, desperta nos acadêmicos sentimentos ambivalentes: "bom" por sentirem-se inseridos na profissão quando iniciam o estágio e "ruim" quando vivenciam a angústia relatada por outros colegas, definida como "Síndrome do 2. ${ }^{\circ}$ Ano"(18). Essas situações vivenciadas simultaneamente provocam uma reação de choque entre o bom e o ruim, gerada principalmente pelo medo e ansiedade desencadeados pela inadaptação do indivíduo frente a uma nova situação $\mathrm{O}^{(8-10)}$.

Os alunos de enfermagem da segunda série sofrem um grande impacto em suas vidas, no primeiro contato com o paciente e, muitas vezes, contato com a morte ${ }^{(19-20)}$.
Acredita-se que, nesse período, o aluno deve receber significativa atenção, quanto à orientação e apoio às dificuldades emocionais, com intuito de prepará-lo para o enfrentamento das possíveis dificuldades que surgem em decorrência desta primeira experiência em campo de estágio hospitalar ${ }^{(19-20)}$. Quanto aos alunos da quarta série, estes sentem-se despreparados para o mercado de trabalho, pois já são praticamente profissionais e sentem medo de atuarem sozinhos sem o apoio do professor ${ }^{(21-22)}$.

$\mathrm{Na}$ primeira e terceira série do curso de graduação, o número de alunos que apresentam sintomas indicativos de depressão é bem menor. Para alguns alunos da primeira série, o começo da vida universitária, é permeado por dúvidas e inseguranças a respeito de sua decisão acadêmica, apresentando questões acerca das possibilidades do mercado de trabalho e do papel profissional a ser desempenhado futuramente ${ }^{(23)}$. Quanto à terceira série, os alunos após passarem pelas dificuldades encontradas na segunda série, acabam sofrendo um processo adaptativo possibilitando que sigam de modo menos sofrido sua construção acadêmica $^{(22,24)}$.

Quanto aos alunos que estudam e trabalham na área da saúde, pode-se perceber que estes apresentaram maior número de pontos de sintomas indicativos de depressão. Conforme a literatura aponta, as pessoas que trabalham e estudam levam uma vida muito intensa e desgastante, podendo apresentar maior número de sintomas indicativos de depressão, devido ao cansaço físico e emocional intensos. A maioria dos alunos que trabalham à noite e estudam pela manhã, têm o sono e sua disposição física afetados, com pouco tempo para estudar e realizar seus trabalhos, além da indisponibilidade para convívio familiar, lazer, necessidades pessoais e dificuldades de conciliarem diversos afazeres ${ }^{(25)}$.

Acreditamos que os alunos que trabalham na área da saúde utilizam mecanismos de defesa para lidarem com suas angústias pessoais como: fragmentação da relação técnico-paciente (ocorre pela divisão do trabalho e para 
diminuir o tempo de contato);despersonalização e negação da importância do indivíduo (a individualidade é negada, inclusive pela vestimenta do paciente e do profissional); distanciamento e negação dos sentimentos (referentes aos sentimentos controlados, envolvimento refreado, identificações perturbadoras); redução do peso da responsabilidade (esta defesa a hierarquia servirá como elemento para diluir e criar soluções facilitadoras, onde irão transitar projeções e fugas dos atos de descuido e imperícia).

\section{CONCLUSÕES}

Os resultados apontam que a população encontra-se, principalmente na faixa etária entre 17 e 20 anos. Representam um grupo na fase final da adolescência, com pouca experiência de vida, que elegeram a enfermagem como profissão sem muitas vezes terem clara a idéia do que isso representa ou o significado para suas vidas. A população estudada merece significativa atenção, uma vez que, dos 119 alunos do Curso de Graduação em Enfermagem, 33 (26,06\%) apresentaram número de pontos de sintomas indicativos de depressão superiores a 20.

Com os resultados obtidos, a implantação de programas de suporte psicológico aos alunos, deve ser prontamente realizada, com o objetivo de dar suporte para lidarem com as situações conflituosas inerentes à vida acadêmica e qualidade de vida pessoal, prevenindo possíveis disfunções e distúrbios.

\section{REFERÊNCIAS}

1. Del Porto JA. Conceito de depressão e seus limites. In: Lafer B, Almeida OP, Fraguas Jr R, Miguel EC. Depressão no ciclo da vida. Porto Alegre: Artes Médicas Sul; 2000. p.20-8.

2. Melo ALN. Psiquiatria. Rio de Janeiro: Guanabara Koogan; 1999. p.30-9.

3. Nogueira- Martins LA. Residência médica: estresse e crescimento. Psychiatry on Line Brazil 1998[citado $2003 \mathrm{Mar}$ 23]; 3(10): [cerca de 11 p.]. Disponível em URL: http:// www.polbr.med.br/arquivo/resid2.htm.

4. Menezes PR, Nascimento AF. Epidemiologia da depressão nas diversas fases da vida. Porto Alegre: Artmed; 2000. p.21-6.

5. Souza JC, Guimarães LAM, Ballone GJ, organizadores. Psicopatologia e psiquiatria básicas. São Paulo: Vetor; 2004. p.25-9.

6. Stuart GW, Laraia MT. Enfermagem psiquiátrica: princípios e prática. 6a. ed. Porto Alegre: Artmed; 2001. p.19-22.

7. Carvalho MDB, Pelloso SM, Valsecchi EASS, Coimbra JAH. Expectativas dos alunos de enfermagem frente ao primeiro estágio em hospital. Rev Esc Enfermagem USP. 1999; 33(2):200-6.

8. Matos SA. Expectativa dos alunos de enfermagem médico cirúrgica frente ao estágio na unidade de centro cirúrgico. Enfoque. 1991; 19: 28-34.

9. OHL RIB. Os sentimentos dos alunos de graduação da área da saúde na primeira experiência formal com o paciente hospitalizado [Monografia de Curso - Especialização em Enfermagem - Saúde Mental e Psiquiátrica]. São Paulo: Escola Paulista de Medicina, Departamento de Enfermagem; 1991. p. 25.

10. Kawakame PMG, Garcia TM. Desvendando o significado de experiências clínicas iniciais de estudantes de graduação em enfermagem. Rev Bras Enfermagem. 2000; 53(3): 355-62.

11. Ângelo M. Elementos componentes do contexto de interações na experiência de aprendizagem prática da aluna de enfermagem. In: Simpósio Brasileiro de Comunicação em Enfermagem, 2. Anais. Ribeirão Preto: Ee-USP - Depto. Enfermagem Geral Especializada; 1990. p.634-47.

12. Jorge MSB. Situações vivenciadas pelos alunos de enfermagem durante o curso, no contexto universitário, apontadas como norteadoras de crises. Rev Esc Enfermagem USP. 1996; 30(1): 138-48.

13. Beck AT, Steer RA, Garbin MG. Psychometric properties of the Beck Depression Inventory: Twenty-five years of evaluation. Clin Psychol Rev. 1988; 8:77-100.

14. Gorenstein C, Andrade LHSG. Inventário de depressão de Beck: propriedades psicométricas da versão em português. Rev Psiquiatr Clin. 1998; 25(5): 245-50.

15. Steer RA, Beck AT, Riskind JH, Brown G. Relationships between the Beck Depression Inventory and the Hamilton Psychiatric Rating Scale for Depression in depressed outpatients. J Psychopathol Behav Assess. 1987; 9(3): 327-39.

16. Kendall PC, Hollon SD, Beck AT, Hammen CL, Ingram RE. Issues and recommendations regarding use of the Beck Depression Inventory. Cognit Ther Res. 1987; 11(3): 289-99.

17. Fiorano AMM. O impacto das emoções no processo ensinoaprendizagem no curso de graduação em Enfermagem [Tese]. São Paulo: Pontifícia Universidade Católica de São Paulo; 2002. p.20-5.

18. Valsecchi EASS, Nogueira MS. Fundamentos de enfermagem: incidentes críticos relacionados à prestação de assistência em estágio supervisionado. Rev Latinoam Enfermagem. 2002; 10(6): 819-24.

19. Carvalho MDB, Pelloso, SM, Valsecchi EASS, Coimbra JAH. Expectativa dos alunos de enfermagem frente ao primeiro estágio. Rev Esc Enfermagem USP. 1999; 33(2): 200-6.

20. Quintana, AM.Cecim PS, Henn CG. O preparo para lidar com a morte na formação do profissional de Medicina. Rev Brás Educ Méd 2002; 26 (03): 21-26.

21. Porcu M, Fritzen CV, Helber C. Sintomas depressivos nos estudantes de Medicina da Universidade Estadual de Maringá [texto na Internet]; [citado 2003 Mar 20]. Disponível em: http://www.unifesp.br/dpsiq/polbr/ppm/ original5_01.htm.

22. Takahashi RT. A opção Profissional do Aluno de Enfermagem: um estudo na Escola de Enfermagem da USP. Nursing (São Paulo). 2001; 4(36): 30-4.

23. 23.Santos TM, Almeida AO, Martins HO, Moreno V . Aplicação de um instrumento de avaliação do grau de depressão em universitários do interior paulista durante a graduação em Enfermagem. Acta Sci Health Sci. 2003; 25(2):171-6.

24. Sánchez RF. Estresores Laborales y Bienestar Psicológico: Impacto en la enfermería hospitalar. Rol Enfermería. 2000; 23:7-8.

25. Pitta AM. Hospital: dor e morte como ofício. 2a ed. São Paulo: Hucitec; 1991. p. 58-77. 


\section{Anexo}

Este questionário consiste em 21 grupos de afirmações. Depois de ler cuidadosamente cada grupo, faça um círculo em torno do número (0, 1, 2 ou 3) diante da afirmação, em cada grupo, que descreve melhor a maneira como você tem se sentido nesta semana, incluindo hoje. Se várias afirmações num grupo parecerem se aplicar igualmente bem, faça um círculo em cada uma. Tome o cuidado de ler todas as afirmações, em cada grupo, antes de fazer a sua escolha.

1. 0 Não me sinto triste.

$1 \mathrm{Eu}$ me sinto triste.

2 Estou sempre triste e não consigo sair disso.

3 Estou tão triste ou infeliz que não consigo suportar.

2. 0 Não estou especialmente desanimado quanto ao futuro. $1 \mathrm{Eu}$ me sinto desanimado quanto ao futuro.

2 Acho que nada tenho a esperar.

3 Acho o futuro sem esperança e tenho a impressão de que as coisas não podem melhorar.

3. 0 Não me sinto um fracasso.

1 Acho que fracassei mais do que uma pessoa comum.

2 Quando olho para trás, na minha vida, tudo o que posso ver é um monte de fracassos.

3 Acho que, como pessoa, sou um completo fracasso.

4. 0 Tenho tanto prazer em tudo como antes.

1 Não sinto mais prazer nas coisas como antes.

2 Não encontro um prazer real em mais nada.

3 Estou insatisfeito ou aborrecido com tudo.

5. 0 Não me sinto especialmente culpado.

1 Eu me sinto culpado às vezes.

2 Eu me sinto culpado na maior parte do tempo.

3 Eu me sinto sempre culpado.

6. 0 Não acho que esteja sendo punido.

1 Acho que posso ser punido.

2 Creio que vou ser punido.

3 Acho que estou sendo punido.

7. 0 Não me sinto decepcionado comigo mesmo.

1 Estou decepcionado comigo mesmo.

2 Estou enojado de mim.

3 Eu me odeio.

8. 0 Não me sinto de qualquer modo pior que os outros.

1 Sou crítico em relação a mim devido a minhas fraquezas ou meus erros.

2 Eu me culpo sempre por minhas falhas.

3 Eu me culpo por tudo de mal que acontece.

9. 0 Não tenho quaisquer idéias de me matar.

1 Tenho idéias de me matar, mas não as executaria.

2 Gostaria de me matar.

3 Eu me mataria se tivesse oportunidade.

10. 0 Não choro mais que o habitual.

1 Choro mais agora do que costumava.

2 Agora, choro o tempo todo.

3 Costumava ser capaz de chorar, mas agora não consigo mesmo que o queira.

11. 0 Não sou mais irritado agora do que já fui.

1 Fico molestado ou irritado mais facilmente do que costumava.

2 Atualmente me sinto irritado o tempo todo.

3 Absolutamente não me irrito com as coisas que costumavam irritar-me.

12. 0 Não perdi o interesse nas outras pessoas.
1 Interesso-me menos do que costumava pelas outras pessoas.

2 Perdi a maior parte do meu interesse nas outras pessoas. 3 Perdi todo o meu interesse nas outras pessoas.

13. 0 Tomo decisões mais ou menos tão bem como em outra época.

1 Adio minhas decisões mais do que costumava.

2 Tenho maior dificuldade em tomar decisões do que antes.

3 Não consigo mais tomar decisões.

14. 0 Não sinto que minha aparência seja pior do que costumava ser.

1 Preocupo-me por estar parecendo velho ou sem atrativos. 2 Sinto que há mudanças permanentes em minha aparência que me fazem parecer sem atrativos.

3 Considero-me feio.

15. O Posso trabalhar mais ou menos tão bem quanto antes.

1 Preciso de um esforço extra para começar qualquer coisa.

2 Tenho de me esforçar muito até fazer qualquer coisa.

3 Não consigo fazer nenhum trabalho.

16. 0 Durmo tão bem quanto de hábito.

1 Não durmo tão bem quanto costumava.

2 Acordo uma ou duas horas mais cedo do que de hábito e tenho dificuldade para voltar a dormir.

3 Acordo várias horas mais cedo do que costumava e tenho dificuldade para voltar a dormir.

17. 0 Não fico mais cansado que de hábito

1 Fico cansado com mais facilidade do que costumava.

2 Sinto-me cansado ao fazer quase qualquer coisa.

3 Estou cansado demais para fazer qualquer coisa.

18. 0 Meu apetite não está pior do que de hábito.

$1 \mathrm{Meu}$ apetite não é tão bom quanto costumava ser.

2 Meu apetite está muito pior agora.

3 Não tenho mais nenhum apetite.

19. 0 Não perdi muito peso, se é que perdi algum ultimamente.

1 Perdi mais de $2,5 \mathrm{Kg}$.

2 Perdi mais de $5,0 \mathrm{Kg}$.

3 Perdi mais de $7,5 \mathrm{Kg}$.

Estou deliberadamente tentando perder peso, comendo menos: $\operatorname{SIM}($ ) NÃO ( )

20. 0 Não me preocupo mais que o de hábito com minha saúde.

1 Preocupo-me com problemas físicos como dores e aflições ou perturbações no estômago ou prisão de ventre.

2 Estou muito preocupado com problemas físicos e é difícil pensar em outra coisa que não isso.

3 Estou tão preocupado com meus problemas físicos que não consigo pensar em outra coisa.

21. 0 Não tenho observado qualquer mudança recente em meu interesse sexual.

1 Estou menos interessado por sexo que costumava.

2 Estou bem menos interessado em sexo atualmente.

3 Perdi completamente o interesse por sexo. 